\title{
The application of pesticides and mineral fertilizers in agriculture
}

\author{
Roman Davydov 1,*, Michael Sokolov², William Hogland ${ }^{3}$, Alexey Glinushkin ${ }^{2}$, and Artem \\ Markaryan ${ }^{4}$ \\ ${ }^{1}$ Peter the Great St.Petersburg Polytechnic University, 195251, Polytechnicheskaya st. 29, St. \\ Petersburg, Russia \\ ${ }^{2}$ All-Russian Research Institute of Phytopathology, 143050, Institute str., ownership 5, Bolshie \\ Vyazemy, Odintsovo distr., Moscow reg., Russia \\ ${ }^{3}$ Linnaeus University, SE-391 82, Kalmar, Sweden \\ ${ }^{4}$ Russian Centre for Science \& Culture, Vester Voldgade 11, 1552 Copenhagen, Denmark
}

\begin{abstract}
Regulation of using pesticides and agrochemicals application in agriculture is one of the important problem now, as also the problem of storage of mineral fertilizers and their improper using, which have negative influence on the chemical and the food security of the country. This paper discusses the features and benefits of monodisperse aerosols of pesticides in plant protection after a long-term research. A new line of development of science, engineering, industrial and innovative technologies - the author's project "Monodisperse Anthropogenic Aerosols" is proposed. The measures for its implementation are presented.
\end{abstract}

\section{Introduction}

Nowadays, about 64-65 thousand tons of plant protection products (pesticides) and more than 2.16 million tons of mineral fertilizers are used in the agricultural production annually. Without the use of crop protection agents, especially chemical, crop production, both in the world and in Russia is impossible. Without the use of the mineral fertilizers, plants are losing their fertility due to exhaustion [1-2].

Analysis of the current situation and possible development scenarios of agricultural production confirms the need for a set of measures for stabilization and restoration of agricultural lands that enhance soil fertility of agricultural lands and improve the overall environmental situation.

The intelligent using of pesticides and agrochemicals connected not only with the expected yields, but also with the safety for humans and animals. It is also important to prevent pesticide contamination of soil and toxic substances (heavy metals, radionuclides) contained in the fertilizer. Another problem is the use of counterfeit, banned or expired pesticides that negatively affect the plants and soil. Falsification of mineral fertilizers - too low content of nutrients compensated excipients containing these toxicants, which leads not only to a shortfall of expected yield, but also to soil contamination. In Fig. 1 the main

\footnotetext{
* Corresponding author: davydovroman@outlook.com
} 
impacts of pesticides on plant, soil and arthropod communities are shown [3]. Orange arrows indicate decreases and blue arrows - increases, empty arrows responsible for indirect effects.

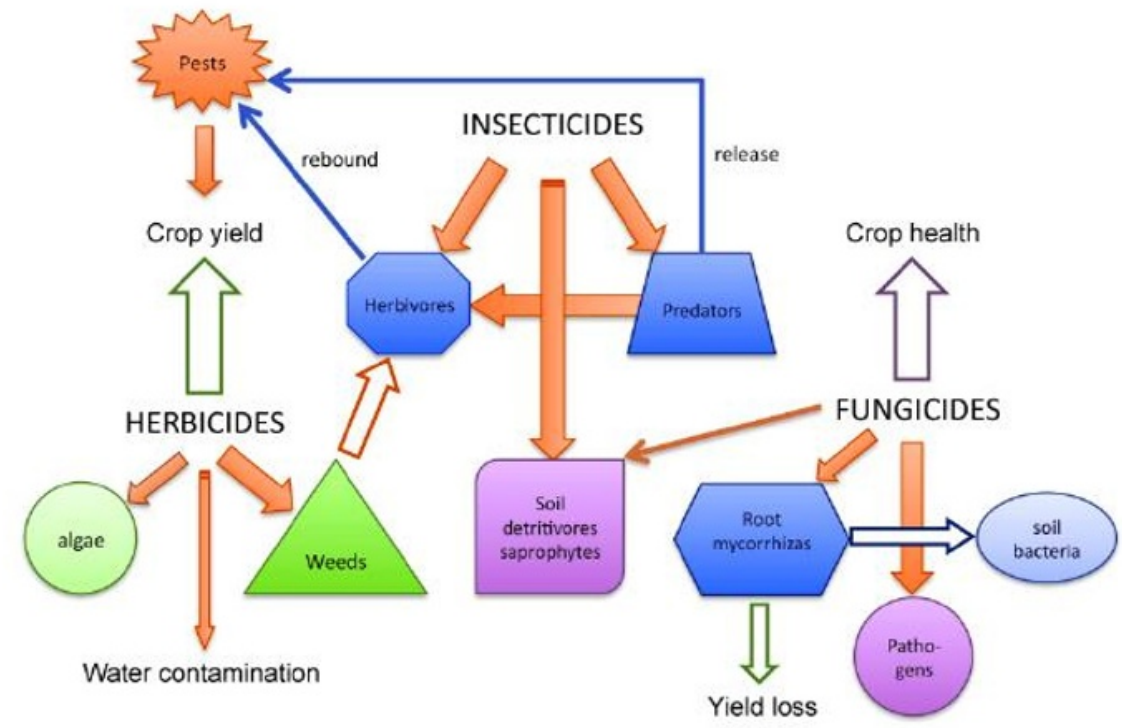

Fig. 1. The scheme of the main impacts of pesticides.

Improper use of pesticides and agricultural chemicals also leads to water pollution and harmful for biological organisms, so the proper use of pesticides and agrochemicals is a key component of the innovative technologies in agricultural production. It should be noted that research on new instruments [4-7] for controlling fertilizers, water bodies and soil is done, but it will take some time to implement the results $[8,9]$.

According to Rosselkhoznadzor the territory of Russia has more than 18 thousand tons of banned and unusable pesticides in various storage conditions. Tons of pesticides (organochlorines, mercury-containing, organophosphorus, including extremely hazardous, relating to the first class of danger) are harmful for human health due to more distant effects of teratogenicity, mutagenicity, carcinogenicity and others. Especially large amounts of unusable and obsolete pesticides accumulated in the Altai Territory, in the Bryansk, Kurgan, Amur, and Smolensk regions in the Primorsky Territory.

According to experts, long-term storage of certain pesticides leads to a significant loss of their hazardous properties. However, this does not apply to persistent organochlorine and mercury-arsenic-containing pesticides. Moreover, some pesticides during prolonged storage and decomposition may form metabolites, which are more dangerous substances to the human health and the environment.

The reduction in the rate of expenditure of pesticides per unit of cultivated area for increasing chemical and biological safety of man and nature - on the one hand and the achievement maximum yields - on the other hand are two global, opposing economic and scientific-technical conflicts of our time. More than half a century, this problem solved only at the expense of corporate progress in the chemistry of pesticides by replacing traditional "hard" pesticides on superplasticity with gram, per-hectare application rates. However, the equipment and the technology of using modern pesticides in the scientific and technical level of the spraying process liquids are same as in second half of the last century (in the standards and there are adjectives such as: "very small, small, medium, large, very large, extremely large." for the range of droplets) [10-12]. 
In the world, hundreds of millions of nozzles and sprayers are working around the clock in various sectors of the economy. However, it is not yet learned to correctly measure the size (volume) of the droplets of the sprayed liquid, the speed and trajectory of their movement and, especially, to optimize their dispersion $[13,14]$. Without a precise definition and regulation of these characteristics, it is impossible to optimize the design of the spray devices to provide a given monodisperse spray for a radical reduction of technological losses of material resources [15-18].

\section{Materials and methods}

One of the main condition of success in establishing energy and resource saving chemical technologies for plant protection is the creation of a monodisperse spray devices for specific chemicals and norms of consumption (solutions, emulsions, suspensions), under specific spraying technique and accordingly, for a specific agricultural technology.

The creation of modern sprayers with adjustable dispersion of the working fluid and their implementation in the system of protection of plants would successfully address the following vital social and environmental objectives:

- To increase the efficiency of pesticide use in agriculture and forestry,

- To eliminate contamination of crops and open water sources, damage and/or destruction of crops and plantings of sensitive crops due to wind drift of herbicides in adjacent areas,

- Significantly reduce ecotoxicant load on agrocenoses, to accelerate their purification,

- To preserve biological diversity and genetic resources of agro-ecosystems,

- To produce normatively clean agricultural products.

The purpose of the research is the improvement of the parameters of spraying the process fluid and objective quantitative control of the parameters of gas-hydrodynamic system. Object of research - torch, gas-hydrodynamic system.

By the experimental methods, mechanics and gas-hydrodynamic effects on the flame spray process fluid is studied. Instruments and experimental techniques - spray actuator, optoelectronic installation with digital signal processing of the image to control the parameters of a disperse medium. The experimental method is studied mechanics and gashydrodynamic effects on the torch of the fluid spray process with disk working body for drops separation. Device mode using herbicides - droplet size in the range 250-350 microns, using insecticides - 160-250 microns, fungicides - 60-160 microns [19-21].

\section{Results and discussion}

The author's patent Method of Estimation of Spray Parameters of Dispersion-Capable Process Fluid and Unit for its Implementation actually begins a new resource-saving scientific and technical branch - "Monodisperse Anthropogenic Aerosols" (MAA) - it is the author's interpretation of a new tendency of development of science engineering, industrial and agricultural technologies [22-25].

The method for estimating the parameters of the fluid spray dispersion-capable process by means of a pulsed laser, the photographic, computer, allowing to sort drops on standard size classes. The most important condition for the MAA project implementation is creation of experimental base. Its task:

a) design and technological assessment of prototypes of monodisperse and polydisperse spray devices and mechanisms;

b) design and development engineering of special test stands.

These stands will provide standard conditions for testing of operation conditions of various nozzles and sprayers used in different fields of science, engineering and production. 
It is planned to equip experimental base with the latest optical and electronic and laser systems for measurement, control and regulation of dispersion degree of the estimated dispersion-fluid systems. For the first time, a tester will be able "to see, measure, create, standardize and reproduce" substance of aerosol droplets spray generated by a sprayer.

Another, no less important priority, scientific and technical task of the MAA Project is creation of modern engineering base or experimental and serial production of monodisperse small and micro-volume chamber and field sprayers. The long-term research carried out in All-Russia Research Institute of Phytopatology showed that monodisperse small and microvolume spraying was bas on the use of conventional or even more cheaper preparative forms of pesticides. The weight of sprayer is significantly reduced due significant reduction of working fluid application rates (and thus, soil compaction is reduced in the process of its work). Productivity is increased and at least $25 \%$ reduction in dose is provided due to elimination of losses of the working fluid in the form of inefficient small and large drops. At the same time, it is important that the testers registered substantial increase of profitability indicators because of protective measures. Thus, decrease in spraying rate of the working fluid flow from 200 to 10 1/ha doubles the productivity.

Due to this, direct costs only for treatment of a crop unit (without regard to pesticides consumption reduction) are reduced by three times. That is significantly better than in similar modern studies of other authors which are used such technics as spinning disc rotary atomizer [26] and spray droplet measurements using shadowgraphy [27]. In addition, there is no need to use new, but not well explored in terms of potential toxicity and not standardized yet, nanopesticides such as nanoemulsions [28] and nanodispesions [29].

Sprayers with adjustable dispersion, enabling to control spraying parameters of the working fluid directly in the field fully comply with international standards of environmental safety - controlled drop application (CDA). However so far, polydisperse fluid spraying causes environmental and economic damage everywhere. Moreover, all this at gigantic scales. We can cope with this problem if we make spraying of working fluids monodisperse and controlled.

\section{Conclusions}

Over-intensification of agriculture without considering the environmental impacts not only leads to soil degradation, but also to the deterioration of the phytosanitary state of crops. The modern innovative system of protection of crops from weeds, pests and diseases should be based on agro-technology and biological components and must lead to a reduction in chemical load on the plants and soil. The long-term research carried out in All-Russia Research Institute of Phytopatology made it possible to improve the parameters of spraying the process fluid and objective quantitative control of the parameters of gas-hydrodynamic system.

\section{References}

1. Y.M. Veretennikov, A.V. Ovsyankina, E.L. Melnikov, I.J. Paremskaya, Repair, restoration, modernization, 10, 48-52 (2013)

2. N.V. Nikitin, Y.Y. Spiridonov, M. Sokolov, Agrochemistry, 11, 51-59 (2008)

3. S.B. Francisco, J.B. Paul, M.M. Reinier, Ecological Impacts of Toxic Chemicals. Bentham Science Publishers, (2011)

4. M. Petrichenko, N. Vatin, D. Nemova, N. Kharkov, A Staritcyna, Applied Mechanics and Materials, 627. 297-303 (2014) 
5. N.S. Myazin, S.E. Logunov, V.V. Davydov, V.Yu. Rud', N.M. Grebenikova, V.V. Yushkova, J. Phys.: Conf. Ser., 929 (1), 012064 (2017)

6. V.V. Davydov, N.S. Myazin, Measurement Techniques, 60 (2), 183-189 (2017)

7. N.S. Myazin, V.V. Davydov, V.V. Yushkova, T.I. Davydova, V.Yu. Rud', J. Phys.: Conf. Ser., 917(4), 042017 (2017)

8. I.S. Kudryashova, V.Yu. Rud', V.Ch. Shpunt, Yu.V. Rud', A.P. Glinushkin, J. Phys.: Conf. Ser., 741, 012106 (2016)

9. V.B. Fadeenko, V.Yu. Rud', Yu.V. Rud', V.Ch. Shpunt, A.P. Glinushkin, N.N. Bykova, J. Phys.: Conf. Ser., 1038(1), 012030 (2018)

10. D.J. Wedlock, Controlled Particle, Droplet and Bubble Formation. Oxford, (2004)

11. D. Tarasova, A. Staritcyna, D. Nemova, K Andreev, MATEC Web of Conf., 53, 01007 (2016)

12. G.A. Matthews, R. Bateman, R. Mille, Pesticide Application Methods, Chichester, (2014)

13. V. Maslak, N. Nasonkina, V. Sakhnoskaya, S. Antonenko, D. Nemova, Procedia Engineering, 117 (1), 985-994 (2015)

14. R.N. Berglund, B.Y. Liu, Environ. Sci. Technol., 7(2), 147-153 (1973)

15. G.A. Mount, J. Am. Mosq. Control Assoc., 14(3), 305-334 (1998)

16. M. Petrichenko, N. Vatin, D. Nemova, N. Kharkov, A Staritcyna, Applied Mechanics and Materials, 627, 297-303 (2014)

17. V. Mushchanov, V. Sievka, A. Veshnevska, D. Nemova, Procedia Engineering, 117, 1018-1026 (2015)

18. Y.M. Veretennikov, I.J. Paremsky, A.V. Ovsyankina, Tractors and agricultural machines, 8, 31-33 (2010)

19. A.P. Glinushkin, O.O. Beloshapkina, A.A. Solovykh, V.S. Lukyantsev, A.A. Batmanova, G.V. Sudarenkov, J. Molnár, Acta Phytopathologica et Entomologica Hungarica, 51(2), 171-181 (2016)

20. M.S. Sokolov, Chemistry in agriculture, 12, 3-10 (1978).

21. A.P. Glinushkin, O.O. Beloshapkina, A.A. Solovykh, G.V. Sudarenkov, J. Molnár, Acta Phytopathologica et Entomologica Hungarica, 51(1), 57-67 (2016)

22. A.M. Semenov, M.S. Sokolov, A.P. Glinushkin, V. I. Glazko, Acta Phytopathologica et Entomologica Hungarica, 52(1), 1-13 (2017)

23. A.O. Terent'ev, I. A. Yaremenko, A. P. Glinushkin, G. I. Nikishin, Russian Journal of Organic Chemistry, 51(12), 1681-1687 (2015)

24. Y.M. Veretennikov, I.J. Paremsky, A.V. Ovsyankina, Tractors and agricultural machinery, 8, 41-44 (2008)

25. Y.M. Veretennikov, V.G. Ostrovsky, P.N. Antoniuk, I.J. Paremsky, A.V. Ovsyankina, Tractors and agricultural machinery, 2, 27-29 (2012)

26. N. Rash, D. Sukhadiya, M. Ojha, S. Tripathi, K. Dashora, International Research Journal of Engineering and Technology, 4(7), 802-810 (2017)

27. G. Matthews, International Pest Control, 58(4), 230-231 (2016)

28. J. Hayles, L. Johnson, C. Worthley, D. Losic, New Pesticides and Soil Sensors, Academic Press, (2017)

29. M.J. Seid, D.J. McClements, Nanoemulsions: Formulation, Applications, and Characterization, Academic Press, (2018) 